\title{
Construction of New Surface Wave Solutions of the Modified KdV Equation
}

\section{Clovis Taki Djeumen Tchaho ${ }^{1,2 *}$, Jean Pierre Ngantcha ${ }^{3}$, Thierry Blanchard Ekogo ${ }^{4,5}$, Bruno Rodin Mbock Um³ , Hugues Martial Omanda ${ }^{1,6}$, Jean Roger Bogning ${ }^{7}$}

\author{
${ }^{1}$ African Centre for Advanced Studies, Yaoundé, Cameroon \\ ${ }^{2}$ Lycée Technique Fulbert Bongotha, Moanda, Gabon \\ ${ }^{3}$ Laboratoire de Mécanique des matériaux, École Polytechnique, Université des Sciences et Techniques de Masuku, Franceville, \\ Gabon \\ ${ }^{4}$ Département de Physique, Université des Sciences et Techniques de Masuku, Franceville, Gabon \\ ${ }^{5}$ Institut International de Recherches Biomédicales et de Biotechnologies Carl Kambangoye (IRBK), Université Internationale de \\ Libreville (UIL), Libreville, Gabon \\ ${ }^{6}$ Laboratoire Pluridisciplinaire des Sciences, École Normale Supérieure, Libreville, Gabon \\ ${ }^{7}$ Department of Physics, Higher Teacher Training College, University of Bamenda, Bamenda, Cameroon \\ Email: ^djeumenclovis@gmail.com, *djeumentchaho@acas-yde.org
}

How to cite this paper: Tchaho, C.T.D., Ngantcha, J.P., Ekogo, T.B., Um, B.R.M., Omanda, H.M. and Bogning, J.R. (2022) Construction of New Surface Wave Solutions of the Modified KdV Equation. Open Journal of Applied Sciences, 12, 196-215. https://doi.org/10.4236/ojapps.2022.122014

Received: December 21, 2021

Accepted: February 18, 2022

Published: February 21, 2022

Copyright $\odot 2022$ by author(s) and Scientific Research Publishing Inc. This work is licensed under the Creative Commons Attribution International License (CC BY 4.0).

http://creativecommons.org/licenses/by/4.0/

\begin{abstract}
The major concern of this work is to propose new prototypes of surface hybrid waves, in particular waves propagating without sprawl or deformation on the surface of a fluid. The model considered for this purpose is the modified KdV (Korteweg-de Vries) equation. A peculiarity of the obtained solutions is that they form packages constituted by combinations of waves belonging to the two main families of well-known bright and dark solitary waves. This putting together creates competitions between the different components of the considered packages which, following the values assigned to the parameters of the considered system and in relation to those of the wave parameters, generate hybrid or multi-form structures. The direct method of resolution which made possible the obtained results is that of Bogning-Djeumen Tchaho-Kofane extended to the new implicit Bogning functions. The existence conditions of some solutions are obtained. The numerical simulations carried out with a view to testing the observable and applicable characters of the obtained solutions revealed their stabilities over a relatively long time, and at the same time, confirmed the recommended theoretical forecasts. We are convinced that the solutions proposed as part of this work will make it possible to detect, understand and explain some physical phenomena linked to fluid molecular interactions, former or new, which constantly occur on the fluid surfaces, mainly at the shallow water surface.
\end{abstract}




\section{Keywords}

Surface Hybrid Waves, Modified Korteweg-De Vries Equation, Bogning-Djeumen Tchaho-Kofané Method, Hybrid Prototypes, Multi-Form Structures

\section{Introduction}

Since its existence, the universe in all its extent and its diversity has made available to human beings, a multitude of physical systems for the most part complex and dynamic, whose understanding of the behavior of these different systems, is more or less beneficial for our progress and our security. These systems in our conception are presented as a domain consisting of an interior environment and an exterior environment both separated by an interface. It, therefore, appears that these three regions are susceptible to many natural or induced phenomena [1]-[10] which in some cases can lead to disasters, thus endangering many lives. Many researchers across the globe have produced works in which several mathematical models [11]-[20], in order to be able to analyze, understand and explain these different phenomena. In the context of this manuscript, we focus our attention on what is happening or could happen at the common border zone between the two domains. This zone is also called interface (or common surface between both domains). Among the different offered models, one of them has long attracted the attention of many researchers, who through multiple works produced, starting from the discovery made by naval engineer John Scott Russel on one day of the year 1834 during the supervision of the works on the Union canal and reported in Report on waves [21] (the solitary wave which he called "Wave of Translation"), passing through Boussinesq (1871), Rayleigh (1876), Kruskal and Zabusky (1965) [1] on the one hand; Gardner, Greene, Kruskal and Miura ([22] [23]) on the other hand and till this day. These works have shed light on the mystery which surrounded Russel's discovery (the emergence of a lone wave in the canal following the abrupt stop of a horse-drawn boat). The said model is the Korteweg-de Vries equation (KdVE) [24] which under its simple form is written [22] [23] [25] [26] [27] [28]

$$
u_{t}+a u u_{x}+u_{x x x}=0 \text {, }
$$

where $a$ is a constant, $u u_{x}$ the Burgers-type nonlinearity term which describes the steepening of the wave, $u_{x x x}$ the third-order dispersion term which accounts for the spreading or dispersion of the wave, and $u_{t}$ characterizes the time evolution of the wave propagating in one direction. Equation (1) is a model which accounts for both dispersion and non-linearity to the lowest order. In this context, one shows that solitons can propagate without strain or sprawl. This equation can admit infinity of solutions of the soliton types [27]. The Korteweg-de Vries (KdV) equation enables to describe the long waves (of long wave- 
length and small amplitude) that propagate on the surface of shallow water, particularly in canals. For more than ten years, surface waves have aroused growing interest in the scientific community. This renewed interest in these waves can be seen through the recent development of metamaterials [29], thus opening up new avenues of application for them [30] [31]. Despite numerous attentions paid to the $\mathrm{KdV}$ equation and despite several numerical and analytical techniques which have made it possible to intensely study solitary waves solutions of this equation, the fact remains that a good number of physical phenomena, observable or not, which occur or could occur on the surface of shallow water, especially in canals, remain unknown and need to be necessarily spotted. Unfortunately, very few, if any, of these works have not alluded to hybrid or multi-form solitary waves that appear as new prototypes of solitary waves of the KdV equation. These solutions once unearthed, will translate new behaviors of the physical systems whose dynamics are described by dispersive and nonlinear equations of the $\mathrm{KdV}$ category. It is within this framework that this work falls in order to provide an adequate response to this shortcoming and to further enrich the literature.

The aim of this work is therefore to use the Bogning-Djeumen Tchaho-Kofané method (BDKm) extended to the implicit Bogning functions (iB-functions) to construct new prototypes of hybrid solitary wave solutions of the modified Korteweg de Vries equation (mKdVE). This is how, in Section 2, we give a brief presentation of the new iB-functions on which the BDKm theory is extended. Section 3 is devoted to the construction of the analytical solutions followed by a numerical study of their stabilities while Section 4 takes care of the useful and necessary discussions for the reader's understanding. Section 5, for its part, concludes this work while highlighting some perspectives.

\section{BDKm Theory}

The $\mathrm{BDKm}$ theory in its latest version is based on the use of $\mathrm{BB}$-functions for the resolution of nonlinear partial differential equations. This use involves mastering the properties of the $\mathrm{iB}$-function in the management and organization of calculations. The iB-functions are defined as [32] [33] [34]

$$
J_{n, m}\left(\sum_{i=0}^{p} \alpha_{i} x_{i}\right)=\frac{\sinh ^{m}\left(\sum_{i=0}^{p} \alpha_{i} x_{i}\right)}{\cosh ^{n}\left(\sum_{i=0}^{p} \alpha_{i} x_{i}\right)}=\sinh ^{m}\left(\sum_{i=0}^{p} \alpha_{i} x_{i}\right) \operatorname{sech}^{n}\left(\sum_{i=0}^{p} \alpha_{i} x_{i}\right) .
$$

The member on the left is the implicit form and the member on the right is the explicit form of the function, where $\alpha_{i},(i=0 ; 1 ; 2 ; \cdots ; p)$ are the parameters associated with the independent variables $x_{i},(i=0 ; 1 ; 2 ; \cdots ; p), m$ is the power of the numerator, $n$ that of the denominator. Thus, these functions are generally used in nonlinear (partial) differential equations of the form

$$
F\left(\phi, \phi_{\xi}, \phi_{\xi \xi}, \cdots,|\phi|^{2}, \phi|\phi|^{2}, \cdots\right)=0,
$$

where $F$ is an arbitrary function which is very often polynomial, $\phi(\xi)$ the unknown function to be determined and $\xi=\sum_{k=0}^{p} \eta\left(x_{k}-v t\right)$ the independent 
variable. The principle consists in building the solution in the form

$$
\phi(\xi)=\sum_{i j} \lambda_{i j} J_{i, j}(\eta \xi)
$$

where $\lambda_{i j}$ are constant coefficients to be determined and $\eta$ a wave parameter. Then, taking into account Equation (4) in Equation (3) landed to the main equation of gamuts

$$
\begin{aligned}
& \sum_{i j n} P_{n}\left(\lambda_{i j}, \eta, v\right) J_{n, 0}(\eta \xi)+\sum_{i j m} Q_{m}\left(\lambda_{i j}, \eta, v\right) J_{m, 1}(\eta \xi)+\sum_{i j k} R_{k}\left(\lambda_{i j}, \eta, v\right) J_{-k, 0}(\eta \xi) \\
& +\sum_{i j l} S_{l}\left(\lambda_{i j}, \eta, v\right) J_{-l, 1}(\eta \xi)+\sum_{i j} Y\left(\lambda_{i j}, \eta, v\right) J_{0,0}(\eta \xi)=0,
\end{aligned}
$$

where $i, j, k, l$ are positive natural integers and $n, m$ the real numbers [32] [33] [34]. Equation (5) is the one from which all the possible analyzes result. The identification at zero of the different coefficients $P_{n}, Q_{m}, R_{k}, S_{l}$ and $Y$ gives rise to series of algebraic equations of unknowns $\lambda_{i j}$ whose order of priority in the resolution is given to the gamut of equations derived from the term $J_{n, 0}(\eta \xi)$, then to those in the terms $J_{m, 1}(\eta \xi), J_{-k, 0}(\eta \xi), J_{-l, 1}(\eta \xi)$ and $J_{0,0}(\eta \xi)$, respectively. The order of precedence here relates to the degree of enrichment and relevance of the series of equations giving the expressions of the coefficients $\lambda_{i j}$ which best approximate the exact values. In the case of the approximate solutions, the resolution of equations coming from the coefficient $P_{n}$ gives satisfaction. For more details, one can refer to the multiple works done in [32]-[51].

\section{Results}

In this section, we first construct the surface hybrid analytical solitary waves, solutions of the modified KdV equation using the BDKm [32]-[51] extended to the new iB-functions. Then, a study of the numerical stability of certain solutions is carried out in order to have a well-established idea of the observable and applicable character of the proposed solutions.

\subsection{Surface Analytical Hybrid Solitary Wave Solutions}

In 1895, the Dutch D. Korteweg and G. de Vries had proposed an equation translating nonlinearity and the dispersion phenomenon. This equation, presented in its simplest form by Equation (1) came to explain the existence of the solitary wave such as observed in 1834 by Russel. The KdV equation is very relevant in the shallow water theory. We consider the KdV equation in a modified form and which is written [24] [26] [27]:

$$
\omega_{t}=\omega_{x x x}+\gamma \omega \omega_{x}+\beta \omega^{2} \omega_{x}
$$

where $\gamma$ and $\beta$ are constant coefficients of the quadratic (the weak nonlinearity) and higher-order cubic nonlinear terms; $\omega_{x x x}$ the dispersion effect term which makes the wave form spread. $t$ and $x$ are both independent temporal and spatial coordinates respectively. $\omega(x, t)$ represents the water's free surface in non-dimensional variables. The competition among the dispersion effect, quadratic and cubic nonlinearities constitute the main interest [52] [53]. For 
some values of the coefficients $\gamma$ and $\beta$, Equation (6) is also called the standard Gardner equation, or the combined $\mathrm{KdV}-\mathrm{mKdV}$ equation. Originally, the Gardner equation was derived rigorously within the asymptotic theory for long internal waves in a two-layer fluid with a density jump at the interface [54]. Equation (6) finds its relevance in various branches of physics, such as quantum field theory, fluid physics, plasma physics and so on. It is also used to model a variety of nonlinear phenomena and to describe internal solitary waves in shallow seas. When we set $\omega(x, t)=\phi(\xi)$, with $\xi=x-v t$ and where $v$ is the wave speed, Equation (6) leads to the traveling wave equation below:

$$
v \phi_{\xi}+\phi_{\xi \xi \xi}+\gamma \phi \phi_{\xi}+\beta \phi^{2} \phi_{\xi}=0,
$$

Since we are looking for hybrid surface solitary wave solutions, these can be chosen after an extension to the new iB-functions as the functions $\phi(\xi)$ of the form:

$$
\phi(\xi)=a J_{2,0}(\eta \xi)+b J_{1,1}(\eta \xi)+c J_{3,0}(\eta \xi)+d J_{2,2}(\eta \xi),
$$

where $a, b, c$ and $d$ are real constants to be determined later, $\eta$, the inverse of the width at half-height of each component of the ansatz given by Equation (8). It is important to point out here that, Equation (8) is a kind of package [32] [33] [34] of solitons whose the first and the third term are bright solitons that differ by their clues and are represented by the iB-functions $J_{2 ; 0}(\eta \xi)$ and $J_{3 ; 0}(\eta \xi)$ respectively; the second term is a kink soliton represented by the $\mathrm{iB}$-function $J_{1 ; 1}(\eta \xi)$, while, the fourth term is a dark soliton and represented by the iB-function $J_{2 ; 2}(\eta \xi)$. To realize this, the reader can simply represent them. This bringing together of the different solitary waves gives a particular character to Equation (8) in the meaning that from their interactions new prototypes of solitary waves emerge that we qualify as hybrids or even multi-form. Let us point out that Equation (8) can be born by Equation (6) as solution. That being said, we continue our analyzes by taking into account Equation (8) in Equation (7). By applying the extended BDKm [32]-[51] to new iB-functions, we obtain the main equation to the coefficients made up of the first two ranges as being:

$$
\sum_{n} P_{n}(a, b, c, d, \gamma, \beta, \eta, v) J_{n, 0}(\eta \xi)+\sum_{m} Q_{m}(a, b, c, d, \gamma, \beta, \eta, v) J_{m, 1}(\eta \xi)=0,
$$

where $n \in\{2 ; 3 ; 4 ; 5 ; 6 ; 7 ; 8\} ; m \in\{3 ; 4 ; 5 ; 6 ; 7 ; 8 ; 9 ; 10\}$. So, Equation (9) has delivered in its formulation, two ranges of equations in the terms of $J_{n, 0}(\eta \xi)$, and $J_{m, 1}(\eta \xi)$, thus constituting the most important ranges according to the BDKm theory. At the same time, this theory also suggests that, in the solving of the series of algebraic equations come from these two ranges, priority is given to the ranges of equations from the coefficients of the terms in $J_{n, 0}(\eta \xi)$ which, in most cases and according to their degree of enrichment in coherent information, suffices to obtain the expressions of the coefficients $a, b, c$ and $d$ as function of parameters $\gamma, \beta, \eta$ and $v$. Further, the investigations carried out in the present case show that the series of equations outcome from the second term of Equation (9) provided only the irrelevant (trivial) results, this in comparison 
with the results provided by equations of the first series (given by the first term of Equation (9). In this context, We should limit ourselves to solving only the equations outcome from terms in $J_{n, 0}(\eta \xi)$. So, the identification at zero, of the coefficients of the terms in $J_{n, 0}(\eta \xi), n \in\{2 ; 3 ; 4 ; 5 ; 6 ; 7 ; 8\}$ results in the series of algebraic equations, with unknowns $a, b, c$ and $d$ as follows

The term in $J_{8,0}(\eta \xi)$,

$$
7 \eta \beta b c^{2}=0,
$$

The term in $J_{7,0}(\eta \xi)$,

$$
12 \eta \beta a b c=0,
$$

The term in $J_{6,0}(\eta \xi)$,

$$
\left(5 \eta \beta a^{2}-2 \eta \beta a d-3 \eta \beta d^{2}-6 \eta \beta c d\right) b=0,
$$

The term in $J_{5,0}(\eta \xi)$,

$$
(4 \eta \gamma-4 \eta \beta a) b c=0
$$

The term in $J_{4,0}(\eta \xi)$,

$$
\left(6 \eta \beta a d+3 \eta \gamma a-2 \eta \beta b^{2}-2 \eta \beta d^{2}-3 \eta \gamma d-4 \eta \beta a^{2}-6 \eta^{3}\right) b=0,
$$

The term in $J_{3,0}(\eta \xi)$,

$$
(-3 \eta \gamma-6 \eta \beta d) b c=0
$$

The term in $J_{2,0}(\eta \xi)$,

$$
\left(\eta v+4 \eta^{3}+3 \eta \gamma d-2 \eta \gamma a+\eta \beta b^{2}+5 \eta \beta d^{2}-4 \eta \beta a d\right) b=0,
$$

We shall not be able to pursue our analyzes without however emphasize the fact that, this series of equations going from Equations (10) to (16) constitutes the key points from which all future analyzes will be born. Thus, on observing the structure of this first series of equations (Equation (10) to Equation (16)), it becomes obvious that the second term of Equation (8) is the most disruptive element of the constituted package [32] [33] [34]. This is justified by the presence of its coefficient $b$ in all the terms of each of the equations of this first series. It is undoubtedly this disturbance which gives rise to the most complex hybrid structures that form in propagation media (see paragraph 3.2. Below). The observation being made, it emerges from Equations (10) and (11) that:

$$
b=0 \text { or } c=0
$$

and

$$
a=0 \text { or } b=0 \text { or } c=0 .
$$

Equations (17) and (18) are those which will guide the choices of the different families of solutions to be constructed. We note in this context that when we set a condition, the major term changes. For example, if $c=0$, Equations (10), (11), (13) and (15) are verify, only Equations (12), (14) and (16) are those to be considered. In the concern of obtaining non-trivial solutions, we shall retain only five families of solutions represented by the following five conditions: 
$a \neq 0, b \neq 0, c \neq 0 ; a \neq 0, b \neq 0, c=0 ; a=0, b \neq 0, c=0 ; a \neq 0, b=0, c=0$ and $a \neq 0, b=0, c \neq 0$.

\subsubsection{First Family of Solutions: Case $a \neq 0, b \neq 0, c \neq 0$}

For $a \neq 0, b \neq 0, c \neq 0$; one obtains from Equations (13) and (15), respectively:

$$
a=\frac{\gamma}{\beta}
$$

and

$$
d=-\frac{\gamma}{2 \beta} .
$$

By substituting Equations (19) and (20) in Equation (12), One obtains:

$$
c=-\frac{7 \gamma}{4 \beta} \text {. }
$$

We continue our investigations by taking into account Equations (19) and (20) into Equation (14), and we reach the results:

$$
b^{2}=-\frac{3 \gamma^{2}+6 \beta \eta^{2}}{2 \beta^{2}},
$$

with the first constraint

$$
\beta \prec-\frac{\gamma^{2}}{2 \eta^{2}} .
$$

The constraint given by Equation (23) shows that, this first family of hybrid solitary waves exists if and only if $\beta \prec 0$ (due to even powers of parameters $\gamma$ and $\beta$ ). So, it is the appearance of the cubic nonlinearity in the propagation medium that is at the origin of the existence of this first new prototype of hybrid solitary waves of the modified $\mathrm{KdV}$ equation. One more time, taking into account Equations (19) and (20) in Equation (16), leads respectively to:

$$
b^{2}=-\frac{\gamma^{2}-16 \beta \eta^{2}-4 \beta v}{4 \beta^{2}},
$$

and the second constraint:

$$
\beta \prec \frac{\gamma^{2}}{16 \eta^{2}+4 v} .
$$

Taking into account Equation (23) in Equation (25) imposes choosing the wave speed according to the third constraint below:

$$
v \prec-4 \eta^{2} \text {. }
$$

It follows from Equation (26) that, the wave speed must remain a lower bound of the singleton $\left\{-4 \eta^{2}\right\}$. So, we can control the speed of the wave from the opposite of the square of the inverse of the width at half-height of each component of the ansatz given by Equation (8), and vice versa. Besides, Equations (23) and (24) must be identical, thus giving rise to the equality: $b^{2}=b^{2}$. Therefore, we derive from this equality, the constraint giving the expression of the parameter 
$\beta$ depending on the parameters $\gamma, \eta$ and $v$ as being:

$$
\beta=\frac{7 \gamma^{2}}{4 \eta^{2}+4 v},
$$

with the aid of Equation (23): $v \prec-\eta^{2}$. In the end, the combination of the constraints given by Equations (23), (25), (26) and (27) imposes the choice of the wave speed $v$ such that:

$$
v \in]-\frac{9}{2} \eta^{2} ;-4 \eta^{2}[.
$$

Equation (22) or Equation (24), taking into account Equation (27) gives, successively:

$$
b^{2}=-\frac{108 \eta^{4}+132 \eta^{2} v+24 v^{2}}{49 \gamma^{2}}
$$

and

$$
b= \pm \sqrt{-\frac{108 \eta^{4}+132 \eta^{2} v+24 v^{2}}{49 \gamma^{2}}} .
$$

Thus, from Equation (8), we obtain the first family of solutions in the form:

$$
\begin{aligned}
\phi(\xi)= & \frac{\gamma}{\beta} J_{2,0}(\eta \xi) \pm \sqrt{-\frac{108 \eta^{4}+132 \eta^{2} v+24 v^{2}}{49 \gamma^{2}}} J_{1,1}(\eta \xi) \\
& -\frac{7 \gamma}{4 \beta} J_{3,0}(\eta \xi)-\frac{\gamma}{2 \beta} J_{2,2}(\eta \xi),
\end{aligned}
$$

with the constraints given by Equations (23), (25), (26), (27) and (28), respectively. It is sound to point out here that, Equation (31) is the main new prototype of the hybrid solitary wave solutions of Equation (6) which is proposed in this work. This new prototype is a package [32] [33] [34] that contain within it several sub-packages within it, notably, the sub-package formed of the first and the second term (consisting of a bright and a kink); the sub-package formed by the second and fourth term (made up of a kink and a dark), the sub-package formed by the first, second and fourth term (made up of a bright, a kink and a dark), just to name a few. It is in this mixing which, during their interactions and according to the taken values by the coefficients and parameters $a, b, c, d, v$ and $\eta$ of the wave and those of the parameters $\gamma, \beta$ of the considered system, generate new hybrid or multi-form structures. It also emerges from Equation (31) that the amplitudes $a, c, d$ of the first, third and fourth term of these wave components are directly proportional to the ratio $X=\frac{\gamma}{\beta}$, which, in their turns means that the taller these wave components the greater the coupled effects of the quadratic and cubic nonlinearity.

\subsubsection{Second Family of Solutions: Case $a \neq 0, b \neq 0, c=0$}

When One sets $c=0$, it follows that Equations (10), (11), (13) and (15) are satisfied, while, Equations (12), (14) and (16) are reduced, successively to: 


$$
\begin{gathered}
5 a^{2}-2 a d-3 d^{2}=0, \\
6 \beta a d+3 \gamma a-2 \beta b^{2}-2 \beta d^{2}-3 \gamma d-4 \beta a^{2}-6 \eta^{2}=0, \\
v+4 \eta^{2}+3 \gamma d-2 \gamma a+\beta b^{2}+5 \beta d^{2}-4 \beta a d=0 .
\end{gathered}
$$

From these three equations, it clearly emerges that Equation (32) is verified if the following condition is satisfied: $a=d$. As a consequence, Equation (33) gives respectively:

$$
b^{2}=-\frac{3 \eta^{2}}{\beta}
$$

and

$$
b= \pm \eta \sqrt{-\frac{3}{\beta}}
$$

with the constraint:

$$
\beta \prec 0 .
$$

Moreover, Equation (34) taking into account Equation (35), leads to the quadratic equation in unknown coefficient $a$ below:

$$
\beta a^{2}+\gamma a+v+\eta^{2}=0 .
$$

Equation (38) has for discriminant:

$$
\Delta=\gamma^{2}-4 \beta\left(v+\eta^{2}\right) .
$$

For $\Delta \geq 0$, Equation (38) admits as solution

$$
a=\frac{-\gamma \pm \sqrt{\gamma^{2}-4 \beta\left(v+\eta^{2}\right)}}{2 \beta},
$$

with $v \leq-\eta^{2}$. It is necessary to point out here that, the case $\Delta \prec 0$ is not necessary because it leads to the complex values of the coefficient $a$, since we are looking for the real solutions. Thus, the second family of solutions of Equation (6), given that: $\cosh ^{2}(\eta \xi)-\sinh ^{2}(\eta \xi)=1$, are the functions $\phi(\xi)$ of the form:

$$
\phi(\xi)=\frac{-\gamma \pm \sqrt{\gamma^{2}-4 \beta\left(v+\eta^{2}\right)}}{2 \beta} \pm \eta \sqrt{-\frac{3}{\beta}} J_{1,1}(\eta \xi),
$$

with the constraints given by Equation (37) and $v \leq-\eta^{2}$. Equation (41) is a prototype that, in its constitution within Equation (8), appeared a priori to be a hybrid structure. But, during the resolution it is formally established that, for this prototype to be a solution of the $\mathrm{mKdV}$ equation considered here, in addition to the fact that $c=0$, it is also necessary that $a=d$. As a consequence, we obtain the solution given by Equation (41) which is a sum of two terms, the first of which is a constant and the second a kink. It becomes easy to note that this second family of solutions can mainly generate only kink and anti-kink structures according to the values taken by the different parameters present in this expression. 


\subsubsection{Third Family of Solutions: Case $a=0, b \neq 0, c=0$}

Under this condition, Equations (10), (11), (13) and (15) are verified. Furthermore, Equation (12) leads to $\beta=0$, while, Equation (14) delivers $d$ in the form

$$
d=-\frac{2 \eta^{2}}{\gamma} \text {. }
$$

The combination of Equations (16) and (42) permits to obtain de following constraint:

$$
v=2 \eta^{2}
$$

for all values of $b$ such as $b \in \mathfrak{R}^{*}$. So, the third family of the solutions of Equation (6) is written:

$$
\phi(\xi)=b J_{1,1}(\eta \xi)-\frac{2 \eta^{2}}{\gamma} J_{2,2}(\eta \xi),
$$

with Equation (43) as constraint, for $\beta=0$ and $b \in \mathfrak{R}^{*}$. Equation (44) is a sub-package derived from the large package given by Equation (8). It is a hybrid prototype that highlights two solitons well-known in the literature, namely the kink represented here by the first and the dark represented by the second term of Equation (44). These two solitons in their interactions give rise to hybrid structures which can be either with strong kink or anti-kink tendencies, or with strong dark or bright tendencies depending on the values taken by the coefficients and parameters $b, d, v$ and $\eta$ of the wave, as well as those taken by the parameters $\gamma$ of the system whose dynamics are governed by Equation (6). Equation (44) also highlights the opposition of signs between the amplitude $d$ and the quadratic nonlinearity parameter $\gamma$ as well as the inversely proportional character of $d$ and $\gamma$ for a given value of $\eta$ of the second term of this equation. This means that, the smaller $\gamma$, the larger $d$ and vice versa. This observation also reflects the antagonistic effect between the two: the taller the wave formed by the second term of Equation (44), the weaker the effect of quadratic nonlinearity and vice versa. This compared to the values taken by $b$ justifies the different hybrid structures formation which appears for $\beta=0$. Thus, this third family of solutions reflects behavior of the considered system and is described by Equation (6) with respect to the phenomena induced by the cubic nonlinearity.

\subsubsection{Fourth Family of Solutions: Case $a \neq 0, b=0, c=0$}

For $a \neq 0, b=0$ and $c=0$, all equations of the first range are verified (from Equation (10) to Equation (16)). The second range leads to a trivial solution which is not important. Thus, according to the implementation of the BDKm, the equations of the first range are the best enriched, thereupon. This makes it possible to extract and retain the approximate solution resulting from this first range for all the values of $a$ and $d$ belonging to the set of non-zero real numbers. We, therefore, obtain the fourth family of solutions of Equation (6) under the form: 


$$
\phi(\xi)=a J_{2,0}(\eta \xi)+d J_{2,2}(\eta \xi),
$$

where $a \in \mathfrak{R}^{*}$ and $d \in \mathfrak{R}^{*}$. Let us note that the sub-package formed by Equation (45) is a hybrid prototype at least under its mathematical form. The structures likely to form oscillate between the bright and the dark solitons depending on the values taken by the various characteristic parameters of the wave and those of parameters that characterize the system whose dynamics are described by Equation (6).

\subsubsection{Fifth Family of Solutions: Case $a \neq 0, b=0, c \neq 0$}

In this context, all equations from (10) to (16) are verified, while, the second range of equations leads to a trivial solution which is not important. So, according to the implementation of the $\mathrm{BDKm}$, the equations of the first range are the best enriched on this subject. This permits to unearth and retain the approximate solution resulting from this first range for all the values of $a, c$ and $d$ belonging to the set of non-zero real numbers. We therefore display the fifth family of solutions of Equation (6) as being:

$$
\phi(\xi)=a J_{2,0}(\eta \xi)+c J_{3,0}(\eta \xi)+d J_{2,2}(\eta \xi),
$$

where $a \in \mathfrak{R}^{*}, c \in \mathfrak{R}^{*}$ and $d \in \mathfrak{R}^{*}$. This fifth family of solutions is a sub-package of which the first two terms are bright solitons and the third term is a dark soliton. Equation (46) is also a hybrid prototype in its mathematical form. Depending on the values taken by parameters of the wave and those of the system whose dynamics is described by Equation (6) mainly generates either bright structures or dark soliton structures.

\subsection{Numerical Simulations of the Obtained Solutions}

This subsection is devoted to numerical simulations aiming to reassure oneself of the observable and applicable characters of certain new hybrid solitary wave prototypes obtained in this work. This should allow laboratories specializing in propagation tests to have the matter to exercise in order to reveal, understand and explain certain new phenomena or behaviors developed by systems whose dynamics are governed by Equation (6). To achieve this, we used certain digital tools, in particular, the MATLAB toolbox pdepe [55] which solves initial-boundary value problems for parabolic-elliptic PDEs in 1-D, with zero flux boundary conditions. We have also used spatially extended grids in others to minimize boundary reflections that could induce spurious effects. It is also necessary to point out here that, these boundary conditions are appropriate to the profiles of the solutions studied in this work, instead of the periodic boundary conditions which require that when a wave passes from one end (of the computational spatial grid) to the other which is opposite to it, it should keep the same properties.

In order to allow a better understand how the above profiles (from Figures 1-3) are obtained, one can illustrate this through an example. Thus, in the case of Figure 1(d): when we set $a=0.00054 ; b=0.0009 ; c=0.0042 ; d=0.004$; $\eta=0.02$, One obtains $\beta=-105.8201 ; \nu=-0.0017$ and $\gamma=-0.2804$ from 


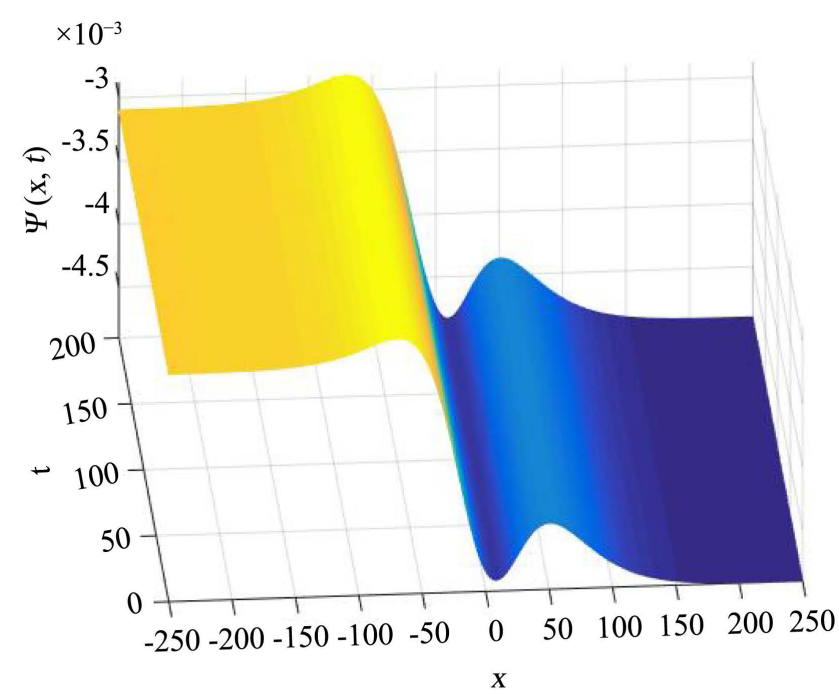

(a)

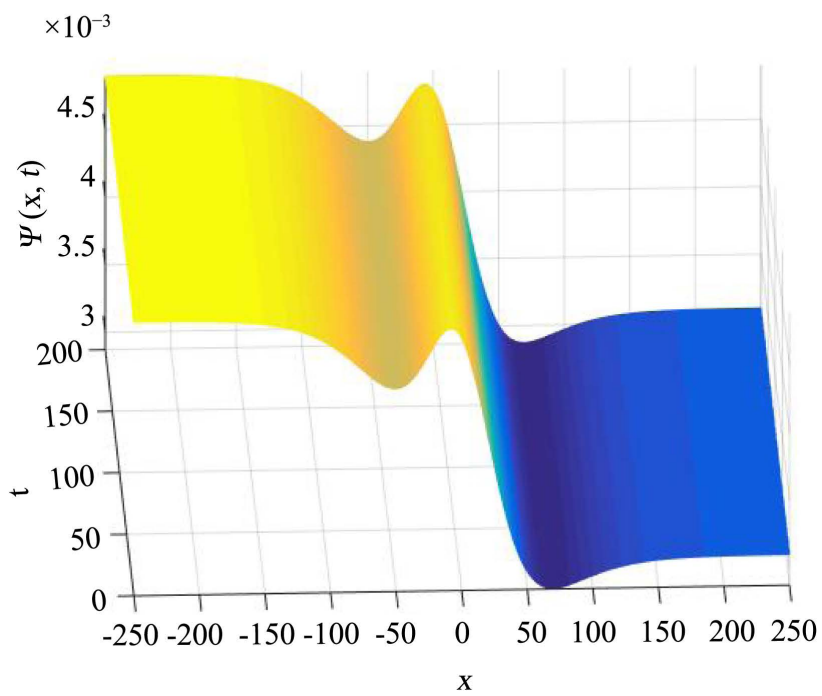

(c)

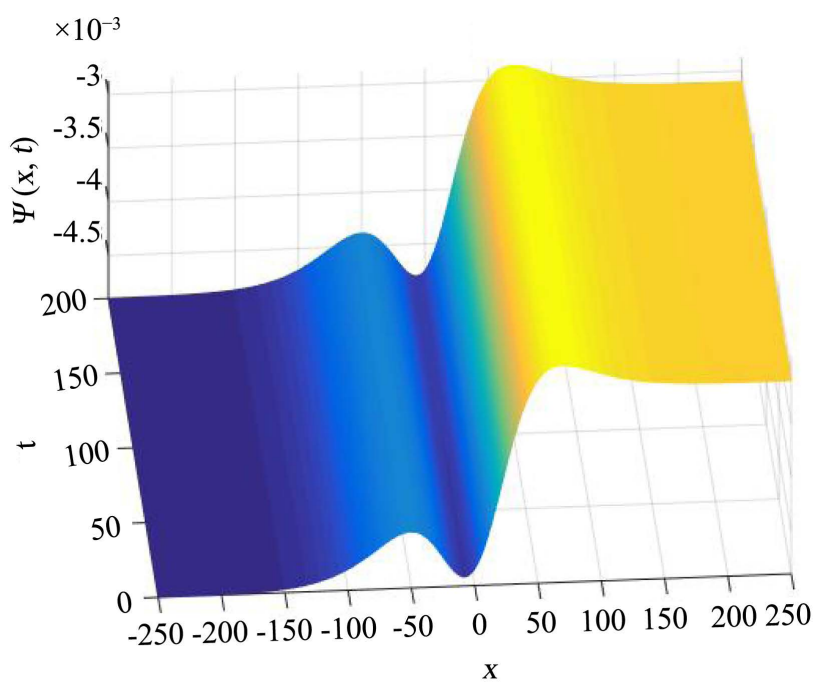

(b)

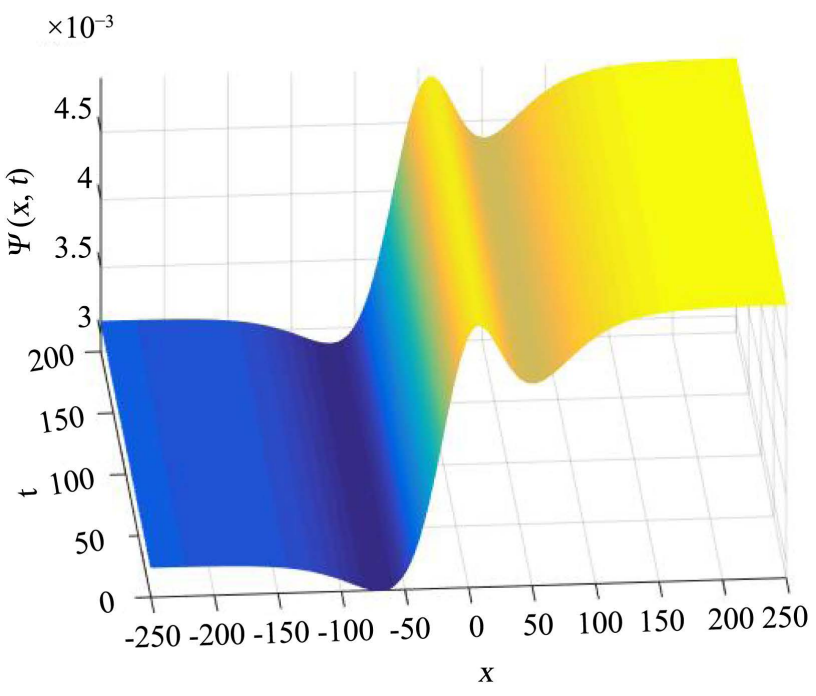

(d)

Figure 1. Robust dynamics of hybrid structures given by Equation (31): (a): Antikink-Bright dynamics: $a=-0.00054$; $b=-0.0009 ; c=-0.0042 ; d=-0.004 ; \eta=0.02 ; v=-0.0017 ; \beta=-105.8201 ; \gamma=-0.2804$. (b): Bright-Kink dynamics: $a=-0.00054 ; \quad b=0.0009 ; \quad c=-0.0042 ; d=-0.004 ; \eta=0.02 ; \quad v=-0.0017 ; \beta=-105.8201 ; \gamma=-0.2804 . \quad$ (c): Dark-Antikink dynamics: $a=0.00054 ; b=-0.0009 ; c=0.0042 ; d=0.004 ; \eta=0.02 ; v=-0.0017 ; \beta=-105.8201$; $\gamma=-0.2804$. (d): Kink-Dark dynamics: $a=0.00054 ; b=0.0009 ; c=0.0042 ; d=0.004 ; \eta=0.02 ; v=-0.0017 ;$ $\beta=-105.8201 ; \gamma=-0.2804$.

Equations (38); (39) and (40), respectively. For more details on the proceedings for obtaining the profiles displayed in the various figures, one can refer to [55]. At the end of the numerical simulations, it appears that Subsection 3.2. has therefore just unveiled some new wave structures likely to appear in physical systems whose dynamics are described by Equation (6). At the same time, they confirmed the made surmises and bearing on the hybrid characters of the wave package [32] [33] [34] represented by Equation (8), thus giving a wider field to the discussions which will follow. 


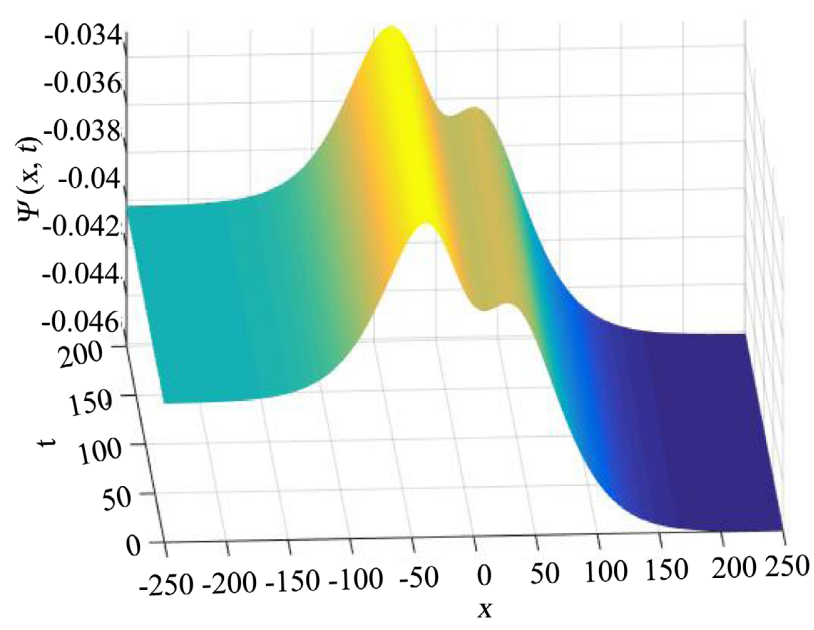

(a)

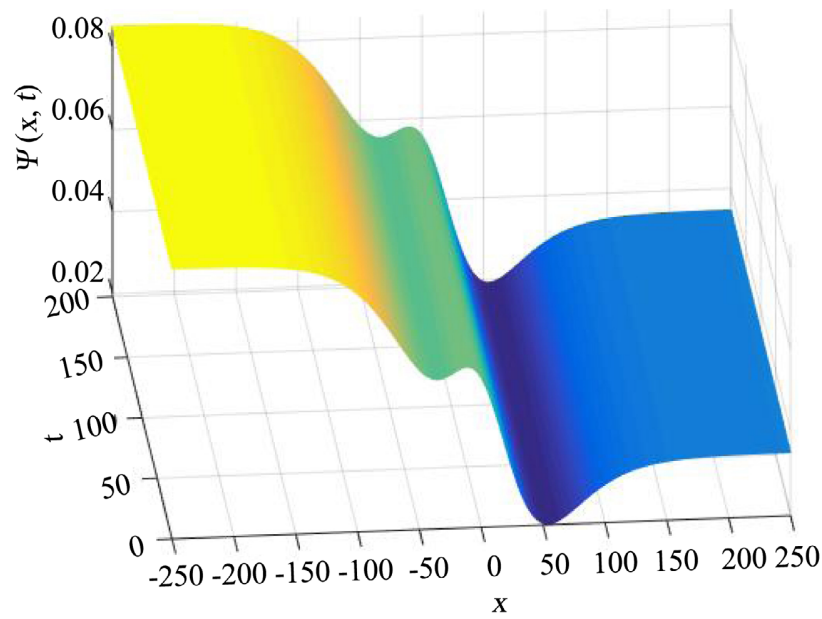

(c)

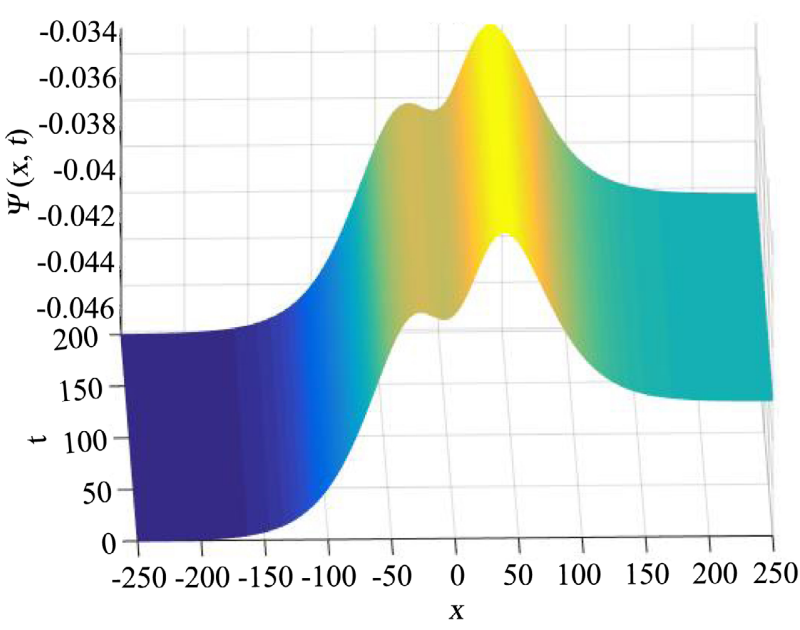

(b)

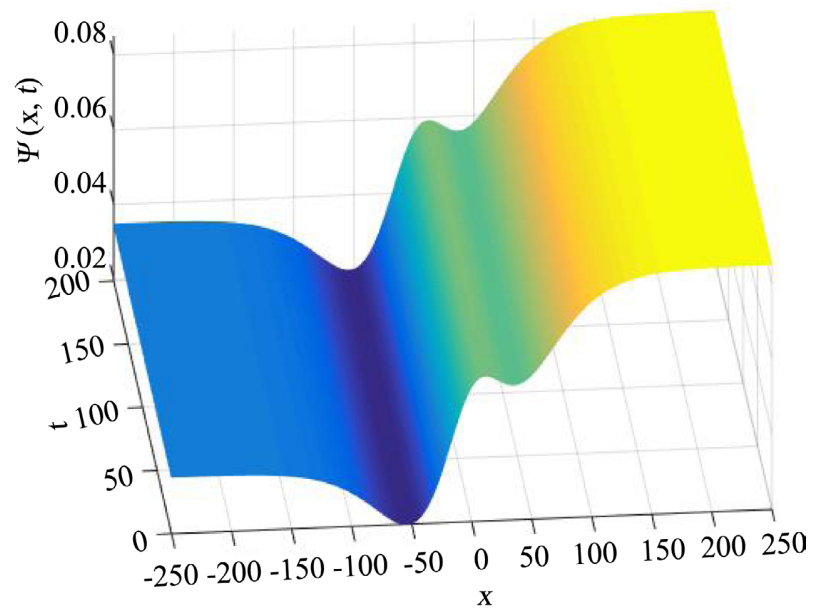

(d)

Figure 2. Robust dynamics of multi-form solitary waves given by Equation (31): (a): Bright-Antikink dynamics: $a=-0.00431$; $b=-0.00292 ; c=-0.0321 ; d=-0.0432 ; \eta=0.02 ; v=-0.0171 ; \beta=-9.0475 ; \gamma=-0.0823$. (b): Kink-Bright dynamics: $a=-0.00431 ; b=0.00292 ; c=-0.0321 ; d=-0.0432 ; \eta=0.02 ; v=-0.0171 ; \beta=-9.0475 ; \gamma=-0.0823$. (c): Antikink-dark evolution: $\eta=0.02 ; \quad v=-0.0017 ; \gamma=0.01 ; a=-0.0743 ; b=-0.0252 ; c=0.13 ; d=0.06 ; \beta=-0.1346$. (d): Dark-kink evolution: $\eta=0.02 ; \quad v=-0.0017 ; \gamma=0.01 ; a=-0.0743 ; b=0.0252 ; c=0.13 ; d=0.06 ; \beta=-0.1346$.

\section{Discussions}

In this part of manuscript, one engages some detailed discussions which help the reader to better appropriate the new ideas offered by the new prototypes of solitary waves obtained and which are the solutions of Equation (6).

Figure 1 shows hybrid prototypes of solitary waves given by Equation (31). The hybridisms displayed here are formal because each of the four profiles integrates undulatory structures of different natures within it. Thus, according to their natures and starting from left to right, we saw fit to name them: (a): Antikink-Bright, (b): Bright-Kink, (c): Dark-Antikink, (d): Dark-Kink, respectively.

A thorough observation of these four profiles reveals at least as regards of their natures, that, Figure 1(a) is the image of Figure 1(c) by a rotation of $\pm \pi$ with respect to an axis parallel to the time axis $t$ and contained in a plane parallel 


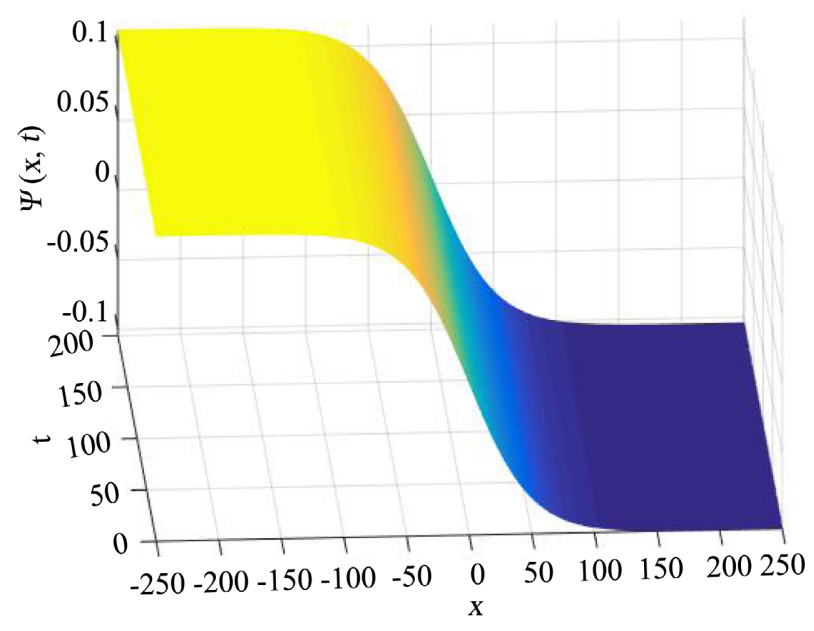

(a)

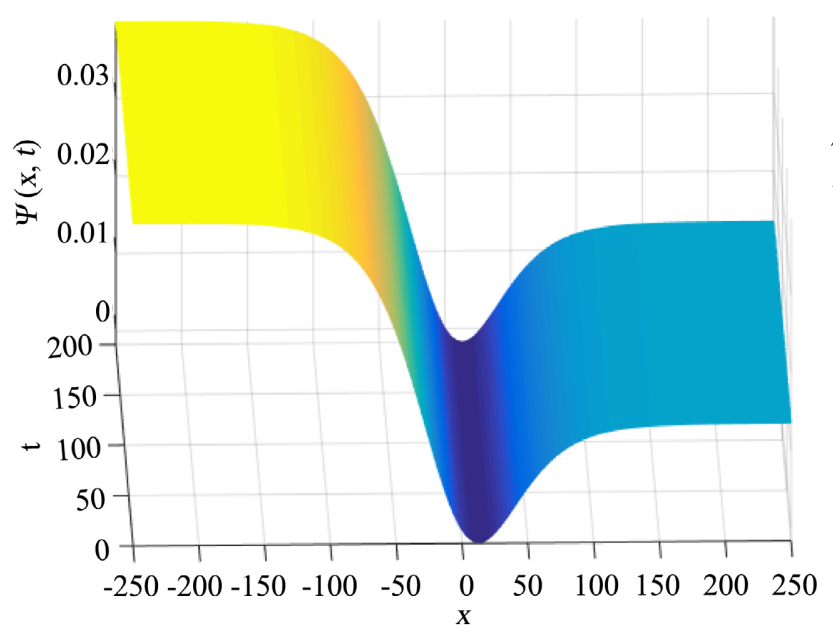

(c)

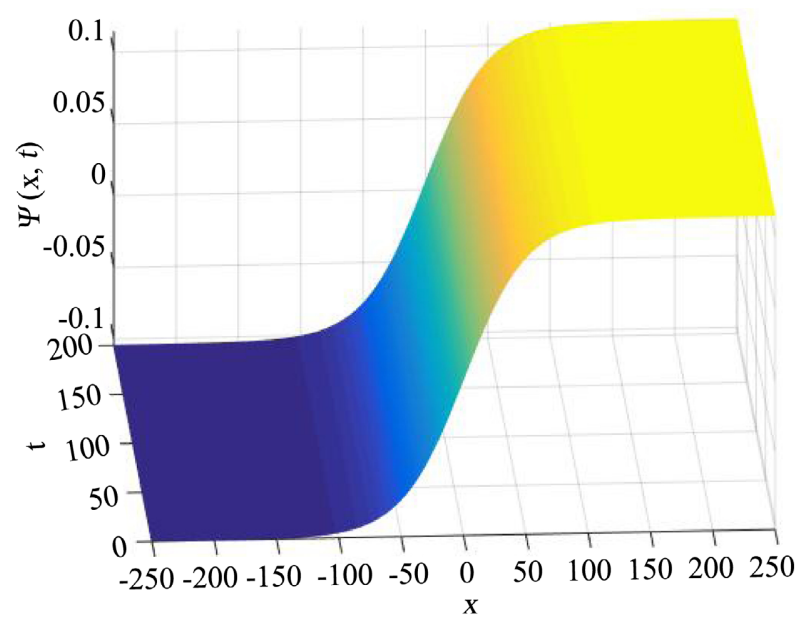

(b)

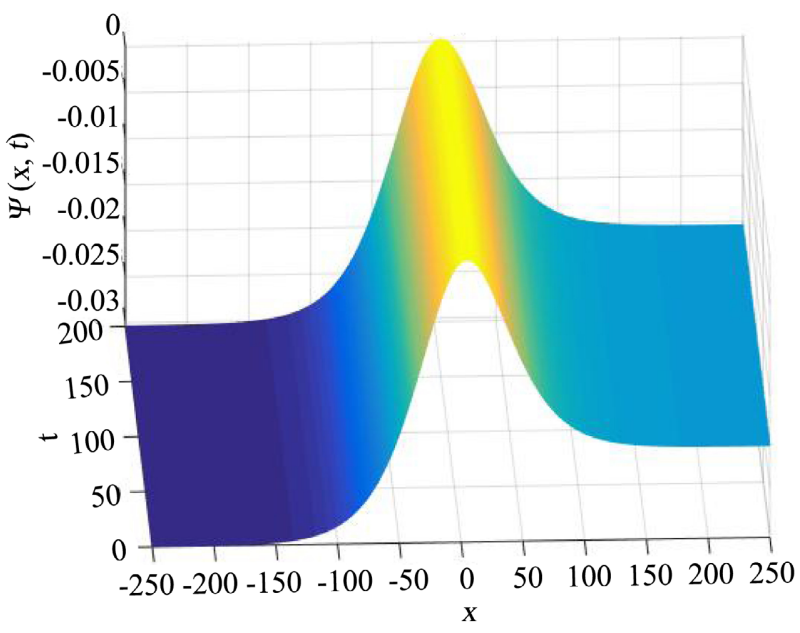

(d)

Figure 3. Stable spatiotemporal evolutions of solitary waves (with some dominant trends) given by Equations (41) and (44): (a): Antikink evolution $\beta=-0.1 ; \gamma=0.02 ; c=0 ; \eta=0.02 ; v=-0.0005 ; a=d=0.0051 ; b=-0.1095$. (b); Kink evolution: $\beta=-0.1 ; \gamma=0.02 ; c=0 ; \eta=0.02 ; v=-0.0005 ; a=d=0.0051 ; b=0.1095$. (c): Antikink-Dark evolution: $a=c=0$; $b=-0.0132 ; \gamma=-0.03 ; \quad \beta=0 ; \eta=0.02 ; v=0.0008 ; d=0.0267$. (d): Kink-Bright evolution: $a=c=0 ; b=0.005$; $\gamma=-0.0315 ; \beta=0 ; \eta=0.02 ; v=0.0008 ; d=-0.0254$.

to the $(x, t)$ plane and vice versa.

It is the same between Figure 1 (b) and Figure 1(d). This transformation will allow a gain in experimentation time during propagation tests with a view to understanding and explaining new phenomena which are direct consequences of the behaviors (translated by these hybrid prototypes of the obtained solitary waves) developed by physical systems whose dynamics are governed by Equation (6).

Moreover, Figure 2 just like Figure 1, displays four formal hybrid profiles which are new solitary wave prototypes obtained. Let us point out here that, the descriptions made in the case of Figure 1 remain valid and should allow the reader to better understand at will the different names attributed to the profiles which Figure 2 emerges. 
Figure 3 depicts on the top row two solitary wave profiles of the same nature: (a) which is an anti-kink and (b) which is a kink, both given by Equation (41). These two profiles thus confirm the a priori ideas made in the case of the second family of solutions of Equation (6). However, the profiles seen on the bottom row are two almost formal hybrid prototypes of solitary waves as one of the structures is predominant. In other words, Figure $3(\mathrm{c})$ is a hybrid prototype which integrates an antikink and a dark, in which the antikink structure is predominant, while Figure 3(d) is also, an almost formal hybrid prototype of solitary wave which also integrate within it a kink and a bright in which the bright structure is predominant. In this context, we estimated the Anti-kink-Dark denomination for (c) and the Kink-Bright denomination for (d). It is important to point out here that the profiles (c) and (d) made it possible to validate the theoretical predictions made as to the predominance of one of the structures which constitute the hybrid prototypes of solitary waves belonging to the third family of solutions of Equation (6) and represented by Equation (44).

After all these discussions, it is clear that numerical simulations revealed many hybrid or multi-form characters of certain obtained solutions, thus, corroborating the theoretical forecasts made on this subject. These results thus confirm the hybrid and novel character, in all its forms (package, sub-packages, and so on.), of the chosen ansatz and represented by Equation (8). Compared to the results obtained in [25] [27] [28], Equation (8) is a new prototype of solitary wave solutions of Equation (6), at least in its analytical form (see Equation (31)), as well as its various new hybrid profiles displayed by Figure 1 and Figure 2 .

\section{Conclusion}

We come to propose at the end of this study, some hybrid prototypes of solitary waves (as well as their existence conditions) of the modified KdV equation depicted by Equation (6). This is made possible thanks to the BDKm extended to the iB-functions, which is very relevant in the resolution of certain types of differential equations which involve dispersive terms and nonlinear terms. The surmises issued on the basic mathematical form (see Equation (8)) from which all the families of the obtained solutions come were validated by numerical simulations carried out with good accuracy [55]. It, therefore, follows that some solitary wave solutions proposed are new and display multi-form characters, in some cases presenting possibilities of rotation which transforms one figure into another (see Figure 1 and Figure 2) and in other cases a predominance of structure (see Figure 3(c) and Figure 3(d)). We believe that a good understanding of the properties of the solutions obtained in the context of this work will allow us to explain certain phenomena that take place on the surface of the domains, in particular on shallow waters surface, at the interface of the two homogeneous fluid media, and so on. More precisely and beyond the mathematical calculations, the choices made on the forms of the constructed solutions are not hazardous. We start from the fact that on the surface of the sea, for example, the 
waves which form and propagate there result from the dynamics of several interacting phenomena. If we remain on the phenomena of collisions, a wave of particular shape observed can be the result of the collision of waves of different natures. Since the main solitary waves that we know are of pulse and kink type, then our approach is clear to know to build hybrid waves resulting from the collision or junction of solitary waves of pulse and kink nature. The objective is to have a fairly robust prototype capable of withstanding even longer instabilities and various fluctuations on the sea surface. This reflection not being isolated can be extended to the case of solid waveguides to also build prototype waves capable of propagating at long distances without amplification. We understand that this study may also be of great interest in information and telecommunications engineering. However, more emphasis should be placed on the permanent spotting of these types of solutions that could help in controlling future phenomena that will take place on the surface of certain physical domains and at the same time will help will help the devise of fluid media whose surface has an improved structure.

\section{Acknowledgements}

The authors' thanks go to both ministries of higher education of Cameroon and Gabon for their assistance through their respective research support programs. These thanks also go to Madame Nchong Glory Arrey who, despite her many occupations, has always been present whenever she has been asked to read this manuscript in order to improve the quality of the English used.

\section{Conflicts of Interest}

The authors declare no conflicts of interest regarding the publication of this paper.

\section{References}

[1] Zabusky, N.J. and Kruskal, M.D. (1965) Interaction of Solitons in a Collisonless Plasma and the Recurrence of Initial States, Physical Review Letters, 15, 240-243. https://doi.org/10.1103/PhysRevLett.15.240

[2] Hirota, R. (1971) Exact Solution of the Korteweg-de Vries Equation for Multiple Collisions of Solitons. Physical Review Letters, 27, 1192-1194. https://doi.org/10.1103/PhysRevLett.27.1192

[3] Fornberg, B. and Whitham, G.B. (1978) A Numerical and Theoretical Study of Certain Nonlinear Wave Phenomena. Philosophical Transactions of the Royal Society, 289, 373-404. https://doi.org/10.1098/rsta.1978.0064

[4] Nohara, B.T. (2003) Derivation and Consideration of Governing Equations of the Envelope Surface Created by Directional, Nearly Monochromatic Waves. Nonlinear Dynamics, 31, 375-392. https://doi.org/10.1023/A:1023266003238

[5] Massel, S.R. (1996) Ocean Surface Waves: Their Physics and Prediction. Tech. Rep., Word Scientific, Singapore. https://doi.org/10.1142/9789812795908

[6] Hossen, M.B., Roshid, H.-O. and Ali, M.Z. (2019) Multi-Soliton, Breathers, Lumps 
and Interaction Solution to the (2+1)-Dimensional Asymmetric Nizhnik-NovikovVeselov Equation. Heliyon, 5, e02548. https://doi.org/10.1016/j.heliyon.2019.e02548

[7] Seadawy, A.R., Nuruddeen, R.I., Aboodh, K.S. and Zakariya, Y.F. (2019) On the Exponential Solutions to Three Extracts from Extended Fifth-Order KdV Equation. Journal of King Saud University-Science, 32, 765-769.

https://doi.org/10.1016/j.jksus.2019.01.007

[8] Hossen, M.B., Roshid, H.-O. and Ali, M.Z. (2018) Charateristics of the Solitary Waves and Rogue Waves with Interaction Phenomena in a $(2+1)$-Dimensional Breaking Soliton Equation. Physics Letters A, 382, 1268-1274. https://doi.org/10.1016/j.physleta.2018.03.016

[9] Hirota, R. (1972) Exact Solution of the Modied Korteweg-de Vries Equation for Multiple Collisions of Solitons. Journal of the Physical Society of Japan, 33, 1456-1458. https://doi.org/10.1143/JPSJ.33.1456

[10] Sugawara, Y., Miyazaki, M. and Li, Y.J. (2020) Surface Potential Measurement by Heterodyne Frequency Modulation Kelvin Probe Force Microscopy in MHz Range. Journal of Physics Communications, 4, Article ID: 075015. https://doi.org/10.1088/2399-6528/aba477

[11] Wazwaz, A.M. and El-Tantawy, S.A. (2016) A New Integrable (3+1)-Dimensional KdV-Like Model with its Multiple-Soliton Solutions. Nonlinear Dynamics, 83, 1529-1534. https://doi.org/10.1007/s11071-015-2427-0

[12] Roshid, H.-O. and Rahman, M.A. (2014) The exp $(-\Phi(\eta))$-expansion Method with Application in the (1+1)-Dimensional Classical Boussinesq Equations. Results in Physics, 4, 150-155. https://doi.org/10.1016/j.rinp.2014.07.006

[13] Seadawy, A.R. and Lu, D. (2017) Bright and Dark Solitary Wave Soliton Solutions for the Generalized Higher Order Nonlinear Schrödingerr Equation and Its Stability. Results in Physics, 7, 43-48. https://doi.org/10.1016/j.rinp.2016.11.038

[14] Medenos, J. and Simões, A. (2001) Heuristic Model for the Energy Spectrum of Phase Turbulence Physical Review E, 64, Article ID: 057301.

https://doi.org/10.1103/PhysRevE.64.057301

[15] Lou, S.Y. (2020) Soliton Molecules and Asymmetric Solitons in three Fifth Order Systems via Velocity Resonance. Journal of Physics Communications, 4, Article ID: 041002. https://doi.org/10.1088/2399-6528/ab833e

[16] Biswas, A., Yildirim, Y., Yasar, E., Zhou, Q., Moshokoa, S.P. and Belic, M. (2018) Optical Solitons for Lakshmanan-Porsezian-Daniel Model by Modified Simple Equation Method. Optik, 160, 24-32. https://doi.org/10.1016/j.ijleo.2018.01.100

[17] Kudryashov, N.A. (2019) General Solution of Traveling Wave Reduction for the Kundu-Mukherjee-Naskar Model. Optik, 186, 22-27. https://doi.org/10.1016/j.ijleo.2019.04.072

[18] Kagan, L. and Sivashinsky, G.I. (2020) Flame Propagation through a Spatially Periodic Shear Flow: Extinction vs. Transition to Detonation. Combustion and Flame, 218, 98-100. https://doi.org/10.1016/j.combustflame.2020.05.008

[19] Nasreen, N., Seadawy, A.R. and Lu, D. (2019) Study of Modulation Instability Analysis and Optical Soliton Solutions of Higher-Order Dispersive Nonlinear Schrödinger Equation with Dual-Power Law Nonlinearity. Modern Physics Letters B, 33, Article ID: 1950309. https://doi.org/10.1142/S0217984919503093

[20] Li, M., Bilige, S., Zhang, R. and Han, L. (2020) Diversity of New Three-Wave Solutions and New Periodic Waves for the $(3+1)$-Dimensional Kadomtsev-PetviashviliBoussinesq-Like Equation. Journal of Applied Mathematics and Physics, 8, 2142-2156. 
https://doi.org/10.4236/jamp.2020.810160

[21] Russell, J.S. (1844) Report on Waves. Report of the Fourteenth Meeting of the British Association for the Advancement of Science.

[22] Gardner, C.S., Greene, J.M., Kruskal, M.D. and Miura, R.M. (1974) Korteweg-de Vries Equation and Generalization. VI. Methods for Exact Solution. Communications in Pure and Applied Mathematics, 15, 97-133. https://doi.org/10.1002/cpa.3160270108

[23] Gardner, C.S., Greene, J.M., Kruskal, M.D. and Miura, R.M. (1967) Method for Solving the Korteweg-de Vries Equation. Physical Review Letters, 19, 1095-1097. https://doi.org/10.1103/PhysRevLett.19.1095

[24] Korteweg, D.J. and de Vries, G. (1895) On the Change of Form of Long Waves Advancing in a Rectangular Canal, and on a New Type of Long Stationary Waves. Philosophical Magazine Series 5, 39, 422-443. https://doi.org/10.1080/14786449508620739

[25] Wazwaz, A.M. (2008) New Sets of Solitary Wave Solutions to the KdV, mKdV, and the Generalized KdV Equations. Communcations in Nonlinear Sciences and $\mathrm{Nu}$ merical Simulations, 13, 331-339. https://doi.org/10.1016/j.cnsns.2006.03.013

[26] Hereman, W. and Nuseir, A. (1997) Symbolic Methods to Construct Exact Solutions of Nonlinear Partial Differential Equations. Mathematics and Computers in Simulation, 43, 13-27. https://doi.org/10.1016/S0378-4754(96)00053-5

[27] Hirota, R. (2004) The Direct Method in Soliton Theory. Cambridge University Press, Cambridge. https://doi.org/10.1017/CBO9780511543043

[28] Wazwaz, A.M. (2009) Partial Differential Equations and Solitary Waves Theory. Higher Education Press, Beijing and Springer-Verlag, Berlin.

https://doi.org/10.1007/978-3-642-00251-9

[29] Smith, D.R., Willie, J., Padilla, D.C., Vier, S.C., Nemat-Nasser and Schultz, S. (2000) Composite Medium with Simultaneously Negative Permeability and Permittivity. Physical Review Letters, 84, 4184-4187. https://doi.org/10.1103/PhysRevLett.84.4184

[30] Petrin, A. (2010) Wave Propagation in Materials for Modern Applications. InTech, Rijeka. https://doi.org/10.5772/113

[31] Baccarelli, R., Burghignoli, P., Frezza, F., Galli, A., Lampariello, P., Lovat, G. and Paulotto, S. (2005) Fundamental Modal Properties of Surface Waves on Metamaterial Grounded Slabs. IEEE Transactions on Microwave Theory and Techniques, 53, 1431-1442. https://doi.org/10.1109/TMTT.2005.845208

[32] Bogning, J.R. (2018) Exact Solitary Wave Solutions of the $(3+1)$ Modified B-Type Kadomtsev-Petviashvili Family Equations. American Journal of Computational and Applied Mathematics, 8, 85-92.

[33] Bogning, J.R. (2019) Mathematics for Nonlinear Physics: The Implicit Bogning Functions and Applications. Lambert Academic Publishing, Saarbrücken.

[34] Bogning, J.R. (2020) Elements of Analytical Mechanics and Quantum Physics. Lambert Academic Publishing, Saarbrücken.

[35] Djeumen Tchaho, C.T., Omanda, H.M., N'tchayi Mbourou, G., Bogning, J.R. and Kofané, T.C. (2021) Higher Order Solitary Wave Solutions of the Standard KdV Equations. Open Journal of Applied Sciences, 11, 103-125. https://doi.org/10.4236/ojapps.2021.111008

[36] Djeumen Tchaho, C.T., Omanda, H.M., N’tchayi Mbourou, G., Bogning, J.R. and Kofané, T.C. (2021) Hybrid Dispersive Optical Solitons in Nonlinear Cubic-Quintic- 
Septic Schrödinger Equation. Optics and Photonics Journal, 11, 23-49. https://doi.org/10.4236/opj.2021.112003

[37] Djeumen Tchaho, C.T. (2015) New Method of Construction of the Solitary Wave Solutions of Some Physical Nonlinear Partial Differential Equations. Doctorat/Ph.D. Thesis, University of Yaounde I, Yaoundé.

[38] Bogning, J.R. (2019) Mathematics for Nonlinear Physics: Solitary Waves in the Center of Resolutions of Dispersive Nonlinear Partial Differential Equations. Dorrance Publishing Co., Pittsburgh.

[39] Ngouo Tchinda, C. and Bogning, J.R. (2020) Solitary Waves and Property Management of Nonlinear Dispersive and Flattened Optical Fiber. American Journal of $O p$ tics and Photonics, 8, 27-32. https://doi.org/10.11648/j.ajop.20200801.13

[40] Djeumen Tchaho, C.T., Bogning, J.R. and Kofané, T.C. (2010) Construction of the Analytical Solitary Wave Solutions of Modified Kuramoto-Sivashinsky Equation by the Method of Identification of Coefficients of the Hyperbolic Functions. Far East Journal of Dynamical Systems, 14, 14-17.

[41] Bogning, J.R., Djeumen Tchaho, C.T. and Kofané, T.C. (2012) Construction of the Soliton Solutions of the Ginzburg-Landau Equations by the New Bogning-Djeumen Tchaho-Kofané Method. Physica Scripta, 85, 25013-25017. https://doi.org/10.1088/0031-8949/85/02/025013

[42] Djeumen Tchaho, C.T., Bogning, J.R. and Kofané, T.C. (2011) Multi-Soliton Solutions of the Modified Kuramoto-Sivashinsky Equation by the BDK Method. Far East Journal Dynamical Systems, 15, 83-98.

[43] Djeumen Tchaho, C.T., Bogning, J.R. and Kofané, T.C. (2012) Modulated Soliton Solution of the Modified Kuramoto-Sivashinsky's Equation. American Journal of Computational and Applied Mathematics, 2, 218-224. https://doi.org/10.5923/j.ajcam.20120205.03

[44] Bogning, J.R., Djeumen Tchaho, C.T. and Kofané, T.C. (2012) Generalization of the Bogning-Djeumen Tchaho-Kofané Method for the Construction of the Solitary Waves and the Survey of the Instabilities. Far East Journal Dynamical Systems, 20, 101-111.

[45] Djeumen Tchaho, C.T., Omanda, H.M. and Belobo Belobo, D. (2018) Hybrid Solitary Waves for the Generalized Kuramoto-Sivashinsky Equation. European Physical Journal Plus, 133, 387. https://doi.org/10.1140/epjp/i2018-12218-4

[46] Djeumen Tchaho, C.T., Omanda, H.M., N’tchayi Mbourou, G., Bogning, J.R. and Kofané, T.C. (2019) Multi-Form Solitary Waves Solutions of the KdV-BurgersKuramoto Equation. Journal of Physics Communication, 3, Article ID: 105013. https://doi.org/10.1088/2399-6528/ab4ba1

[47] Njikue, R., Bogning, J.R. and Kofané, T.C. (2018) Exact Bright and Dark Solitary Wave Solutions of the Generalized Higher-Order Nonlinear Schrödinger Equation Describing the Propagation of Ultra-Short Pulse in Optical Fibers. Journal of Physics Communication, 2, Article ID: 025030. https://doi.org/10.1088/2399-6528/aaaf3b

[48] Bogning, J.R., Djeumen Tchaho, C.T. and Omanda, H.M. (2016) Combined Solitary Wave Solutions in Higher-Order Effects Optical Fibers. British Journal of Mathematics \& Computer Science, 13, 1-12. https://doi.org/10.9734/BJMCS/2016/10620

[49] Bogning, J.R., Fautso Kuiaté, G., Omanda, H.M. and Djeumen Tchaho, C.T. (2015) Combined Peakons and Multiple-Peak Solutions of the Camassa-Holm and Modified KdV Equations and Their Conditions of Obtention. Physics Journal, 3, 367-374.

[50] Bogning, J.R., Porsezian, K., Fautso Kuiaté, G. and Omanda, H.M. (2015) Gap Solitary Pulses Induced by the Modulational Instability and Discrete Effects in Array of 
Inhomogeneous Optical Fibers. Physical Journal, 1, 216-224.

[51] Omanda, H.M., N'tchayi Mbourou, G., Djeumen Tchaho, C.T., Bogning, J.R. and Kofané, T.C. (2021) Kink-Bright Solitary Wave Solution of the Generalized Kuramoto-Sivashinsky Equation. Far East Journal of Dynamical Systems, 1, 59-80. https://doi.org/10.17654/DS033010059

[52] Wazwaz, A.M. (2007) New Solitons and Kink Solutions for the Gardner Equation. Communications in Nonlinear Sciences and Numerical Simulations, 12, 1395-1404. https://doi.org/10.1016/j.cnsns.2005.11.007

[53] Wazwaz, A.M. (2007) New Solitons and Kinks Solutions to the Sharma-Tasso-Olver Equation. Applied Mathematics and Computational, 188, 1205-1213.

https://doi.org/10.1016/j.amc.2006.10.075

[54] Slyunaev, A.V. and Pelinovski, E.N. (1999) Dynamics of Large-Amplitude Solitons. Journal of Experimental Theoritical Physics, 89, 173-181. https://doi.org/10.1134/1.558966

[55] Skeel, R.D. and Berzins, M. (1990) A Method for the Spatial Discretization of Parabolic Equations in One Space Variable. SIAM Journal on Scientific and Statistical Computing, 11, 1-32. https://doi.org/10.1137/0911001 